\title{
Hormonal manipulation in androgen signaling: a narrative review on using novel androgen therapy agents to optimize clinical outcomes and minimize side effects for prostate cancer patients
}

\author{
Yung Lyou, Tanya B. Dorff^ \\ Department of Medical Oncology and Experimental Therapeutics, City of Hope National Medical Center, Duarte, CA, USA \\ Contributions: (I) Conception and design: All authors; (II) Administrative support: TB Dorff; (III) Provision of study materials or patients: None; (IV) \\ Collection and assembly of data: None; (V) Data analysis and interpretation: None; (VI) Manuscript writing: All authors; (VII) Final approval of \\ manuscript: All authors. \\ Correspondence to: Tanya B. Dorff. Department of Medical Oncology and Experimental Therapeutics, City of Hope National Medical Center, Duarte, \\ CA, USA. Email: tdorff@coh.org.
}

\begin{abstract}
Prostate cancer is a significant public health burden and one of the most common cancers globally and in the United States. The current cornerstone of prostate cancer systemic treatment involves the suppression of androgen receptor (AR) signaling, either by reducing the body's testosterone production or inhibiting its binding to $\mathrm{AR}$ and its subsequent gene regulatory network driving carcinogenesis. This signaling pathway plays a central role in both hormone sensitive and castration resistant prostate cancer (CRPC), as evidenced by survival benefit when AR-targeted therapies are applied in the setting of CRPC. With the development of increasingly potent central and peripherally acting androgen targeting agents physicians treating prostate cancer can expect to treat their patients for a longer duration with a larger selection of effective agents. In this setting clinicians are now faced with questions of how to best tailor treatments for the prostate cancer patient to not only maximize overall survival but also optimize the quality of life and mitigate toxicity. In this manuscript we discuss the newer hormone therapy agents for prostate cancer and highlight what they indicate about optimizing medical castration, and the potential value of peripheral blockade.
\end{abstract}

Keywords: Central androgen blockade; peripheral androgen blockade; prostate cancer; androgen signaling

Submitted Jun 30, 2020. Accepted for publication Dec 01, 2020.

doi: $10.21037 /$ tau-20-1053

View this article at: http://dx.doi.org/10.21037/tau-20-1053

\section{Introduction}

Prostate cancer is a significant public health burden and one of the most common cancers amongst men both in the United States and globally $(1,2)$. It is estimated that in the year 2020 there will be approximately 193,000 new cases and 33,000 deaths attributed to prostate cancer in the United States (2). The current cornerstone of prostate cancer systemic treatment involves the suppression of androgen receptor (AR) signaling, either by reducing the body's testosterone production (central androgen blockade) or inhibiting its binding to $\mathrm{AR}$ and its downstream signaling in prostate cancer cells (peripheral blockade) as depicted in Figure 1. The central role of AR signaling driving prostate cancer was first reported nearly 80 years ago in 1941 by Drs. Charles Huggins and Clarence Hodges (3). These two investigators showed that androgens promoted prostate cancer growth and by inhibiting the amount of circulating

^ ORCID: 0000-0001-5990-298x. 


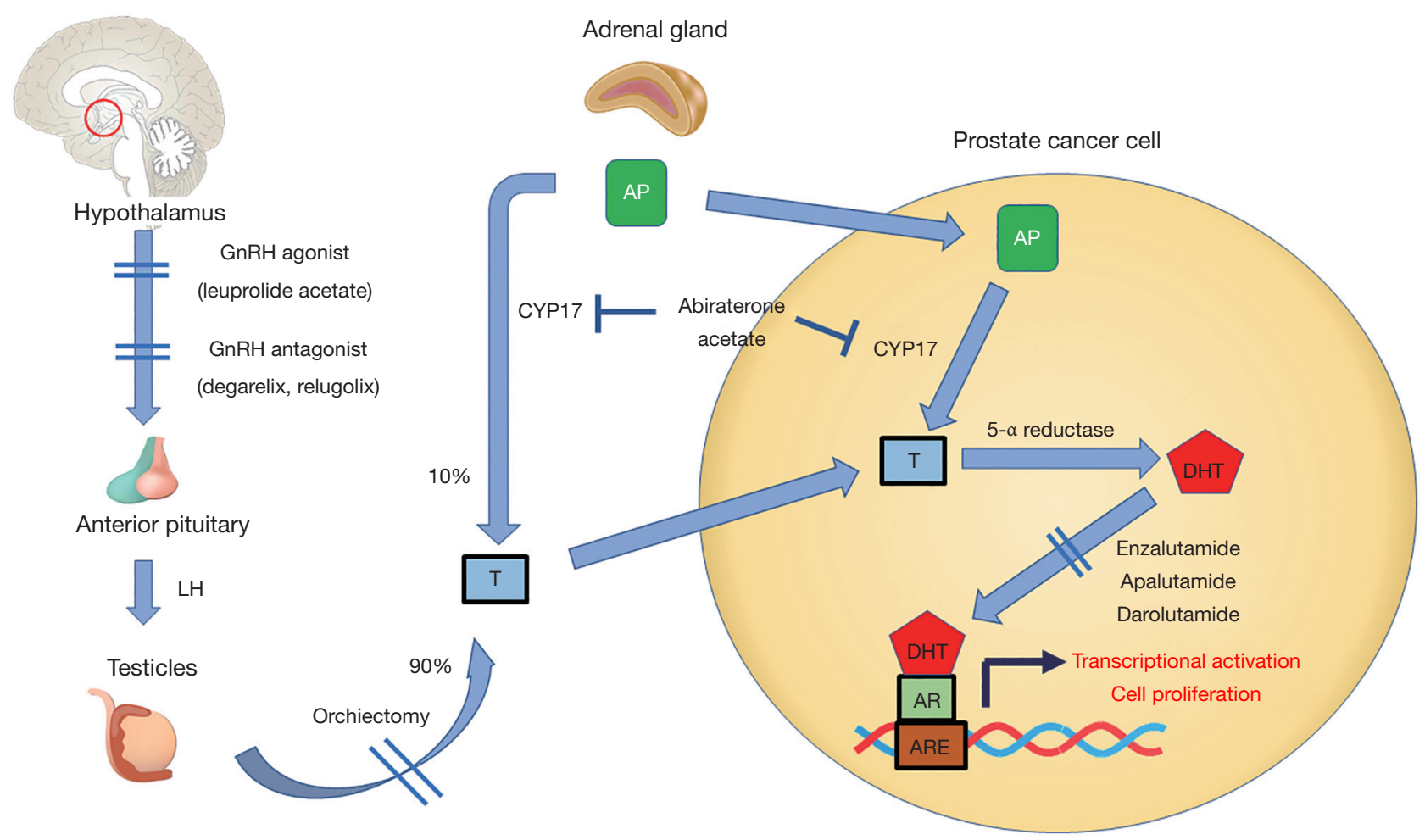

Figure 1 Androgen blockade in prostate cancer treatment. Central androgen blockade is accomplished through the use of GnRH targeting agents or surgical orchiectomy. Peripheral androgen blockade is done through the use of antiandrogen agents such as abiraterone acetate, enzalutamide, apalutamide, or darolutamide. AP, androgen precursor, T, testosterone, AR, androgen receptor, ARE, androgen response element, DHT, dihydrotestosterone.

androgens through the use of bilateral orchiectomy or exogenous estrogens one could palliate the symptoms of bone metastases in patients with advanced disease $(3,4)$. The Nobel prize was later awarded to Dr. Charles Huggins for this seminal discovery that would later lead to the development of today's modern androgen deprivation therapy (ADT) regimens used in the treatment of prostate cancer. We present the following article in accordance with the NARRATIVE REVIEW reporting checklist (available at http://dx.doi.org/10.21037/tau-20-1053).

\section{Manipulation of central AR signaling by targeting the pituitary-gonadal axis}

The initial approach to ADT was eliminating testicular production of androgens by performing a bilateral orchiectomy. Although this approach is highly effective, and is still utilized in low resource settings, the use of medical castration by central androgen blockade using gonadotropin releasing hormone $(\mathrm{GnRH})$ agonists and antagonists reduces some psychological barriers such as body image change, and provides the potential for reversibility (5). A summary of central acting ADT agents is provided in Table 1. A meta-analysis of 10 independent studies involving 1908 patients done by Seidenfeld et al. found that the overall survival in prostate cancer patients who received medical castration (i.e., GnRH agonist or antagonist) or surgical orchiectomy was not significantly different (6). Furthermore, a recent randomized prospective study $(\mathrm{n}=58)$ done by Østergren $e t a l$. where patients were randomized to surgical orchiectomy or medical castration through the use of triptorelin (GnRH agonist) found that medical castration was able to achieve superior testosterone reduction (7). Overall, these results suggested that medical castration is an effective alternative to surgical orchiectomy and has become the de-facto standard, whereas surgical castration remains a valid alternative option.

Testosterone suppression induced by $\mathrm{GnRH}$ agents or orchiectomy may not be complete due to the potential for ongoing testosterone production from sources outside 
Table 1 Comparison of central androgen blockade agents

\begin{tabular}{|c|c|c|c|c|}
\hline Name of agent & Dosing duration & Method of delivery & Common adverse effects & Pharmacologic category \\
\hline $\begin{array}{l}\text { Degarelix } \\
\text { (Firmagon }^{\circledR} \text { ) }\end{array}$ & 4 weeks & $\begin{array}{l}\text { Subcutaneous depot } \\
\text { injection }\end{array}$ & $\begin{array}{l}\text { Injection site reaction }(35 \%) \text {, fever } \\
(1-10 \%) \text {, chills }(5 \%)\end{array}$ & $\begin{array}{l}\text { Gonadotropin-releasing } \\
\text { hormone antagonist }\end{array}$ \\
\hline
\end{tabular}

of the testicles, most importantly the adrenal glands. Furthermore, the clinical significance of inadequate testosterone suppression and of testosterone kinetics such as the "flare" effect of GnRH agonists has become clearer, leading to increased emphasis on testosterone monitoring during castration therapy (8). A randomized phase 3 clinical trial comparing degarelix (the first GnRH antagonist) to leuprolide ( $\mathrm{GnRH}$ agonist) found that degarelix was non-inferior in suppressing testosterone to $\leq 0.5 \mathrm{ng} / \mathrm{mL}$ at all monthly measurements in $97.2 \%$ and $96.4 \%$ of patients respectively (9). Furthermore, it was found that testosterone levels reached desired range in $96.1 \%$ of degarelix patients by 3 days and none in the leuprolide group (9). PSA declines at 14 days were achieved by $64 \%$ of degarelix patients compared to $18 \%$ of leuprolide patients, and at 28 days there was still a difference ( $85 \%$ for degarelix, $68 \%$ for leuprolide). Interestingly, though many feel the initial loading dose to immediately drop testosterone is the most important advantage of GnRH antagonism, in the cross-over experience, men on leuprolide who crossed over to degarelix when study results were reported had a significant improvement in PSA progression free survival than those who stayed on leuprolide, suggesting that optimal ongoing suppression is relevant $(9,10)$.

GnRH agonists such as leuprolide and goserelin remain widely used due to several advantages, including depot dosing and lack of injection site reaction. Injection site reactions occur in $40 \%$ of patients receiving degarelix, and manifests as redness, tenderness, and occasionally with fever and chills (9). Degarelix's high rate of injection site reactions and its monthly dosing have limited widespread adoption of this agent. However, there are reports identifying improved cardiac safety with degarelix. In a phase 3 clinical trial, degarelix was associated with similar rates of significant QT prolongation, but ischemic heart disease occurred at a much lower rate at $4 \%$ of men treated with degarelix compared to $10 \%$ of men treated with leuprolide (11). Mechanistically, it has been difficult to explain why there would be a difference in cardiovascular toxicity since low testosterone levels are felt to be a major contributor to the cardiovascular morbidity associated with androgen deprivation. Some preclinical work suggests FSH levels play a role, and have found that destabilization of existing atherosclerotic plaques is greater with leuprolide than with degarelix (12). Additionally, differences in the constellation of metabolic changes induced by degarelix were noted in mouse models, with more visceral fat deposition and weight gain seen after orchiectomy or leuprolide compared to degarelix (13). The ongoing PRONOUNCE study will provide direct prospective evidence in a randomized comparison of leuprolide and degarelix with measurement of hsCRP and other cardiovascular risk biomarkers at baseline and during the trial to better understand the associated cardiovascular risks in men receiving either of these treatment with pre-existing atherosclerotic cardiovascular disease (14).

Just recently FDA approved, the first orally administered GnRH antagonist, relugolix, reported superior testosterone suppression compared to leuprolide in the phase 3 HERO trial (15). There was also more rapid testosterone recovery after discontinuation relugolix. In this study major adverse cardiac events (defined as myocardial infarction, central nervous system hemorrhages, deaths due to all causes, and ischemic heart disease) were reported to be lower in the relugolix group. This study also added (protocol amendment 3) exploratory blood testing for biomarkers of cardiovascular disease or ischemia. The exploratory blood analysis of cardiovascular disease biomarkers has not yet been reported and will be of interest to see if it can provide further biological insight into understanding the differences of cardiovascular risk between these two agents. Pending additional information, such as any relevant drug-drug interactions, relugolix may become a preferred option for 
patients with a history of, or at high risk for, major cardiac events and when rapid testosterone recovery after a course of therapy is desired. There is some concern about how the high compliance seen during the clinical trial may be difficult to replicate in routine clinical practice and could result in some variability in testosterone suppression in a real-world patient population. Another variable that remains unknown is how this agent will compare in terms of cost effectiveness to degarelix or leuprolide. If there is a higher financial burden on the patient or overall healthcare system this may impact widespread adoption and utilization of this agent.

For now, differences in cardiovascular toxicity should not be the sole determinant of which medical castration agent is utilized, as the benefits in patients with advanced prostate cancer of these agents tend to outweigh the associated cardiovascular risk. Nevertheless, it is important for physicians to consider cardiovascular comorbidity and to discuss with patients what is known about cardiovascular risk and GnRH therapy. Overall, these agents have similar efficacy and toxicity profiles, with some differences as outlined above. Both GnRH agonists and antagonists represent effective treatments in the wide variety of disease states in which medical castration has been shown to be beneficial. As discussed above, current clinical practice in delivering central androgen blockade for prostate cancer requires the monitoring of serum testosterone levels during treatment to ensure that medical castration is being achieved and vigilantly evaluating the patient's overall medical status to minimize drug toxicity or switch agents if needed.

\section{Manipulation of cellular AR signaling: the AR antagonists and CYP17 inhibitors}

Prostate cancer cells eventually develop resistance to central androgen blockade via multiple mechanisms, two of which are alterations in the AR (mutations, amplification) and intracellular testosterone production via upregulation of CYP17. Increasing PSA despite castrate levels of testosterone defines the transition from castration sensitive prostate cancer (CSPC) to the castration resistant prostate cancer (CRPC) state, heralding the lethal phase of the disease. Even after the development of castration resistance, AR signaling remains central to cancer progression in this phase, as evidenced by the strong success of agents targeting these two resistance mechanisms: blocking production of testosterone by adrenals and cancer cells (i.e., abiraterone acetate), and the AR binding/translocation (i.e., apalutamide, darolutamide, and enzalutamide). The newer AR antagonists, and the CYP17 inhibitor abiraterone acetate, have all been developed and approved for use in conjunction with castration therapy.

Given that abiraterone acetate could conceivably suppress all sources of testosterone production, both testicular and extragonadal, the question arises whether it could be used as monotherapy. During early phase clinical trials of abiraterone acetate, the levels of testosterone suppression were evaluated at escalating doses of abiraterone; at doses of $500 \mathrm{mg}$, testosterone levels decreased by $50 \%$ on the second day, but did not consistently reach target castrate levels, and recovery to normal levels was noted by 6-9 days due to increasing luteinizing hormone (LH) levels (16). At the $800 \mathrm{mg}$ dose, target castrate levels of testosterone were maintained in 2 of 3 subjects, despite the increased LH (16). Acknowledging the limited data, it appears that monotherapy with abiraterone acetate does not achieve sustained or consistent testosterone suppression and cannot therefore be utilized in lieu of central androgen blockade. In addition, abiraterone acetate's ability to block the testosterone flare with GnRH agonist therapy has not yet been studied. Overall there is insufficient evidence at present to suggest that abiraterone acetate monotherapy is sufficient to deliver fully effective androgen blockade, and thus it should be used in combination with central androgen blockade agents (i.e., GnRH agonists or antagonists).

\section{Peripheral androgen blockade through the use of AR antagonists in prostate cancer}

While AR antagonists were developed to be utilized in conjunction with castration, the question has arisen whether suppression of androgen signaling at the cellular level, while maintaining serum levels of testosterone, could be effective. Interest in a peripheral blockade, or AR antagonist monotherapy, approach has been fueled by patients' desire to reduce hypogonadal side effects such as hot flashes, loss of muscle, decreased libido, and erectile dysfunction (17). Studies of AR antagonist efficacy as monotherapy have largely been performed as single-arm phase II studies in advanced prostate cancer, which limits clinical utility. An early peripheral blockade study (CALGB 9782), which administered flutamide plus dutasteride, was notable for a relatively high rate of toxicity-18\% or subjects discontinued treatment due to diarrhea, gynecomastia, and transaminitis (18). 
A meta-analysis done by Kunath et al. attempted to address the question of peripheral blockade by combining these smaller studies ( $\mathrm{n}=11)$ involving 3,060 patients across a variety of prostate cancer stages treated with $A R$ antagonist monotherapy published before the year 2013 (17). Although definite conclusions could not be drawn due to the heterogeneous quality of the included studies, including major variability in duration of follow-up, the meta-analysis suggested that peripheral androgen blockade was associated with less favorable prostate cancer control (17). Contrary to what was expected, side effects were not necessarily more favorable with peripheral blockade, and more antiandrogen monotherapy patients stopped treatments due to side effects, which included notably breast tenderness, gynecomastia, and generalized fatigue (17). Patient receiving central androgen blockade did report more hot flashes, nocturia, and decreased libido (17). Overall, the authors concluded that there was not a sufficient level of evidence to suggest that peripheral androgen blockade could achieve similar overall survival in advanced prostate cancer patients, and recommended against its use without more rigorous prospective clinical trial data (17).

The meta-analysis by Kunath et al. predated the FDA approval of better tolerated and more effective second-generation AR antagonists such as apalutamide, enzalutamide, and darolutamide. As a result, the question has resurfaced as to whether these newer agents would be better tolerated and/or effective enough to be utilized as monotherapy in prostate cancer. Enzalutamide monotherapy was studied in 67 men with CSPC and $92.5 \%$ of patients had $>80 \%$ PSA decline at the 25 -week timepoint, indicating strong activity (19). Durability of response appeared to be good, with $62.7 \%$ of men remaining on therapy at the 3 -year timepoint (20). The finding that $61.9 \%$ of men had PSA $0.1 \mathrm{ng} / \mathrm{mL}$ or less compares favorably with the CSPC intensification trials such as LATITUDE with abiraterone added to up-front castration therapy $(19,21)$. However, the enzalutamide monotherapy study is difficult to contextualize, since it included patients with non-metastatic prostate cancer and had a limited number of subjects $(\mathrm{n}=67)(19)$. Although these results were encouraging, they will need to be validated in a larger prospective clinical trial. Like the enzalutamide monotherapy study discussed above, there are similar ongoing and completed phase II studies with apalutamide and darolutamide which have yet to report their results.

The LACOG-0415 trial (NCT02867020) reported at the 2020 ASCO Annual Meeting was a prospective randomized phase 2 clinical trial that compared combination $\mathrm{ADT}$ and abiraterone acetate vs. apalutamide monotherapy $v s$. apalutamide with abiraterone acetate. This trial yielded important direct comparative evidence of how peripheral androgen blockade performed relative to castration with a primary endpoint of PSA nadir at week 25 . $\mathrm{ADT}$ and abiraterone acetate as well as apalutamide plus abiraterone acetate both performed better than apalutamide monotherapy (20). In both combination arms testosterone was suppressed while serum testosterone levels increased with apalutamide monotherapy. Despite this increase in serum testosterone levels, quality of life was not improved on the apalutamide monotherapy arm. In fact, it was noted that more men on the apalutamide monotherapy arm complained of gynecomastia while the other arms had greater likelihood of hot flashes. For now, the use of AR antagonists as monotherapy has not shown a clear advantage either in efficacy or toxicity, and thus remains investigational and best undertaken as part of a clinical trial.

\section{Androgen targeted agents with salvage radiation for biochemically recurrent prostate cancer}

When PSA rises after radical prostatectomy salvage radiation is applied with curative intent. Recently large randomized trials indicate an advantage to the addition of ADT during salvage radiation $(22,23)$. In the RTOG 9601 trial, high dose bicalutamide $(150 \mathrm{mg})$ administered for 2 years increased overall survival from $71.3 \%$ to $76.3 \%$ at 12 years with a hazard ratio of 0.77 (22). The GETUG-AFU 16 study utilized GnRH therapy for 6 months and found an improvement in its primary end point of 120 -month progression-free survival from $49 \%$ to $64 \%$ with hazard ratio 0.54 [HT $0.54(0.43-0.68)$, stratified log-rank test $\mathrm{P}<0.0001]$ (23). Metastasis-free at 120 -months was also found to be improved from $69 \%$ to $75 \%$ in patients who received radiotherapy alone versus radiotherapy plus ADT with HR of 0.73 (0.54-0.98; stratified log-rank test $\mathrm{P}=0.0339$ ) (23). Overall survival which was a secondary end point was not found to be significantly improved (23). Based on the results of the GETUG-AFU 16 and also the SPPORT trial, which has been presented in abstract form only, the addition of ADT through GnRH therapy to salvage radiotherapy remains highly utilized given these positive findings of PFS and MFS as surrogate endpoints for OS $(23,24)$.

There are ongoing investigations into if the second-generation antiandrogens, apalutamide and 
enzalutamide, could be utilized in conjunction with radiotherapy for biochemical recurrence due to more potent inhibition of AR signaling and evidence from in vitro studies of radiosensitization properties (25). However, in addition to efficacy, toxicity must be considered and it should not be assumed that these agents will spare patients from some traditional ADT toxicities. For example, enzalutamide in the monotherapy study was found to lead to modest declines in global health status and sexual activity at 3 years using the EORTC QLQ-C30 quality of life tool (26). Bone density was relatively less impacted; decreases of $0.1 \%$ to $2.7 \%$ were noted, which compares favorably with literature-reported decreases of $1.4-7.6 \%$ for central androgen blockade using medical castration $(27,28)$. The SALV-ENZA trial is a phase II randomized placebo-controlled trial comparing salvage radiation with enzalutamide or placebo for 6 months in men with biochemical recurrence of prostate cancer after radical prostatectomy (NCT02203695) with the primary endpoint of biochemical recurrence free survival. Another study, the SAVE trial (NCT03899077), will randomize 202 men with biochemical recurrence to salvage radiation with apalutamide or with a $\mathrm{GnRH}$ agonist/antagonist with the primary endpoint of changes in sexual function. These, and other, large prospective randomized studies will be crucial in establishing the relative risks and benefits to a castration versus peripheral blockade approach during salvage radiation.

\section{Duration of ADT during intermittent therapy for biochemically recurrent prostate cancer}

In the setting where salvage local therapy has failed or is not an option, intermittent androgen deprivation therapy is the standard of care. This is typically GnRH based therapy, with variable duration. Since the JPR7 study found no advantage for continuous ADT (29) while quality of life was better for men treated intermittently, most patients and physicians favor intermittent therapy in this setting. The fact that overall survival was the same, with more prostate cancer deaths on the intermittent arm and more cardiovascular deaths on the continuous arm, suggests that longer duration of ADT increases cardiovascular risk. However, changes in cardiac risk factors, such as HbA1c and low-density lipoprotein levels, have been shown to be impacted as early as 12 and 24 weeks respectively $(30,31)$. The literature on cardiovascular risk and ADT has yielded inconsistent assessments, and thus minimizing duration of
ADT can only be hypothesized to improve cardiovascular risk, with more prospective data needed to better clarify this association.

The ongoing intensification study AFT-19 selected a control arm of 1 year with GnRH agonist or antagonist, to which the experimental arms add apalutamide or apalutamide and abiraterone (NCT03009981). However, whether there is a need to treat for a full year is called into question by the findings of a study of duration of treatment during intermittent degarelix for biochemically recurrent prostate cancer (32). In this phase II study 4 months of degarelix was compared to 10 months of degarelix monotherapy (32). It was found that there was no difference in time off treatment before reaching the re-treatment PSA level between these two groups (32). While this was a small study $(\mathrm{n}=90)$ and the re-treatment trigger was different than in JPR7 (PSA $\geq 5 \mathrm{ng} / \mathrm{mL}$ ) it does highlight the lack of data, and potentially the inadequacy of a "one size fits all" approach with timed intervention and fixed PSA triggers. Treating to undetectable nadir, when possible, represents one way to tailor the approach to the individual patient. Furthermore, in practice the PSA level at which treatment cycles are triggered can be based on not only absolute PSA value, but pre-prostatectomy PSA, rate of PSA rise, and PET imaging-this type of individualization has been difficult to create in the clinical trial setting and could impact outcomes.

\section{Taking into account side effects in the era of having choices: non-metastatic CRPC}

Three large randomized phase 3 clinical trials with very similar designs (PROSPER = enzalutamide, SPARTAN $=$ apalutamide, ARAMIS $=$ darolutamide) have each shown that the adding a second-generation $\mathrm{AR}$ antagonist to castration in non-metastatic CRPC can significantly improve the metastasis-free survival endpoint and delay the development of symptomatic visible metastatic lesions (33). However, as these were all separate independent trial there is no way to distinguish the efficacy or discriminate a difference in target population, and side effects may become the determining factor in selecting an agent for these patients.

Darolutamide was designed with a unique chemical structure compared to apalutamide and enzalutamide, to avoid activating mutant $\mathrm{AR}$, but which also prevents the drug from crossing the blood-brain barrier (34). This could impact cognitive toxicities and seizure risk, which are 
toxicities seen with apalutamide and enzalutamide. Because there are no directly comparative trials, a definitive answer to this question is not yet available, but the adverse event profile of darolutamide was closer to placebo in its trial than the other agents were to placebo in their respective trials (33). Prospective studies are ongoing to clarify the clinical significance with longitudinal detailed cognitive assessments.

\section{Conclusions}

Despite its long history androgen suppression via both central and peripheral blockade remains a core principle in the treatment of advanced prostate cancer. Thanks to the development of many new effective therapeutic agents the overall survival for these patients is now measured in the multitude of years. Therefore, it has become more imperative than ever before that we refine our treatments to not only maximize overall survival but also optimize the quality of life while minimizing toxicity in these patients. By understanding the efficacy and toxicity profile of each of these agents and monotherapy versus combination therapy approaches, the treating clinician can choose the optimal strategy for each patient's overall medical profile.

\section{Acknowledgments}

Funding: Research reported in this publication included work performed at the City of Hope, supported by the National Cancer Institute of the National Institutes of Health (P30CA033572). The content is solely the responsibility of the authors and does not represent the official views of the National Institutes of Health.

\section{Footnote}

Provenance and Peer Review: This article was commissioned by the Guest Editors (Badrinath R. Konety, Daniel W. Lin) for the series "Current and Future Topics on Prostate Cancer" published in Translational Andrology and Urology. The article has undergone external peer review.

Reporting Checklist: The authors have completed the NARRATIVE REVIEW reporting checklist. Available at http://dx.doi.org/10.21037/tau-20-1053

Conflicts of Interest: Both authors have completed the ICMJE uniform disclosure form (available at http://dx.doi. org/10.21037/tau-20-1053). The series "Management of Advanced Genitourinary Malignancies" was commissioned by the editorial office without any funding or sponsorship. TBD reports personal fees from Janssen, personal fees from Bayer, personal fees from Abbvie, outside the submitted work. The authors have no other conflicts of interest to declare.

Ethical Statement: The authors are accountable for all aspects of the work in ensuring that questions related to the accuracy or integrity of any part of the work are appropriately investigated and resolved.

Open Access Statement: This is an Open Access article distributed in accordance with the Creative Commons Attribution-NonCommercial-NoDerivs 4.0 International License (CC BY-NC-ND 4.0), which permits the noncommercial replication and distribution of the article with the strict proviso that no changes or edits are made and the original work is properly cited (including links to both the formal publication through the relevant DOI and the license). See: https://creativecommons.org/licenses/by-nc-nd/4.0/.

\section{References}

1. Bray F, Ferlay J, Soerjomataram I, et al. Global cancer statistics 2018: GLOBOCAN estimates of incidence and mortality worldwide for 36 cancers in 185 countries. CA Cancer J Clin 2018;68:394-424.

2. Siegel RL, Miller KD, Jemal A. Cancer statistics, 2020. CA Cancer J Clin 2020;70:7-30.

3. Huggins C, Hodges CV. Studies on Prostatic Cancer. I. The Effect of Castration, of Estrogen and of Androgen Injection on Serum Phosphatases in Metastatic Carcinoma of the Prostate. Cancer Res 1941;1:293-7.

4. Huggins C. Studies on prostatic cancer: II. The effects of castration on advanced carcinoma of the prostate gland. Arch Surg 1941;43:209.

5. Boccon-Gibod L. Optimising Hormone Therapy in Advanced Disease. Eur Urol Suppl 2005;4:21-9.

6. Seidenfeld J, Samson DJ, Hasselblad V, et al. Single-therapy androgen suppression in men with advanced prostate cancer: a systematic review and meta-analysis. Ann Intern Med 2000;132:566-77.

7. Østergren PB, Kistorp C, Fode M, et al. Luteinizing Hormone-Releasing Hormone Agonists are Superior to Subcapsular Orchiectomy in Lowering Testosterone Levels of Men with Prostate Cancer: Results from a Randomized 
Clinical Trial. J Urol 2017;197:1441-7.

8. Morote J, Orsola A, Planas J, et al. Redefining clinically significant castration levels in patients with prostate cancer receiving continuous androgen deprivation therapy. J Urol 2007;178:1290-5.

9. Klotz L, Boccon-Gibod L, Shore ND, et al. The efficacy and safety of degarelix: a 12-month, comparative, randomized, open-label, parallel-group phase III study in patients with prostate cancer. BJU Int 2008;102:1531-8.

10. Crawford ED, Shore ND, Moul JW, et al. Long-term tolerability and efficacy of degarelix: 5-year results from a phase III extension trial with a 1-arm crossover from leuprolide to degarelix. Urology 2014;83:1122-8.

11. Smith MR, Klotz L, Persson BE, et al. Cardiovascular safety of degarelix: results from a 12-month, comparative, randomized, open label, parallel group phase III trial in patients with prostate cancer. J Urol 2010;184:2313-9.

12. Knutsson A, Hsiung S, Celik S, et al. Treatment with a $\mathrm{GnRH}$ receptor agonist, but not the $\mathrm{GnRH}$ receptor antagonist degarelix, induces atherosclerotic plaque instability in ApoE-/- mice. Sci Rep 2016;6:26220.

13. Hopmans SN, Duivenvoorden WCM, Werstuck GH, et al. GnRH antagonist associates with less adiposity and reduced characteristics of metabolic syndrome and atherosclerosis compared with orchiectomy and $\mathrm{GnRH}$ agonist in a preclinical mouse model. Urol Oncol 2014;32:1126-34.

14. Melloni C, Slovin SF, Blemings A, et al. Cardiovascular Safety of Degarelix Versus Leuprolide for Advanced Prostate Cancer. JACC Cardio Oncol 2020;2:70-81.

15. Shore ND, Saad F, Cookson MS, et al. Oral Relugolix for Androgen-Deprivation Therapy in Advanced Prostate Cancer. N Engl J Med 2020;382:2187-96.

16. O’Donnell A, Judson I, Dowsett M, et al. Hormonal impact of the 17alpha-hydroxylase/C(17,20)-lyase inhibitor abiraterone acetate (CB7630) in patients with prostate cancer. Br J Cancer 2004;90:2317-25.

17. Kunath F, Grobe HR, Rücker G, et al. Non-steroidal antiandrogen monotherapy compared with luteinizing hormone-releasing hormone agonists or surgical castration monotherapy for advanced prostate cancer: a Cochrane systematic review. BJU Int 2015;116:30-6.

18. Monk JP, Halabi S, Picus J, et al. Efficacy of peripheral androgen blockade in prostate cancer patients with biochemical failure after definitive local therapy: Results of Cancer and Leukemia Group B (CALGB) 9782. Cancer 2012;118:4139-47.

19. Scher HI, Fizazi K, Saad F, et al. Increased survival with enzalutamide in prostate cancer after chemotherapy. N Engl J Med 2012;367:1187-97.

20. Werutsky G, Maluf FC, Cronemberger EH, et al. The LACOG-0415 phase II trial: abiraterone acetate and $\mathrm{ADT}$ versus apalutamide versus abiraterone acetate and apalutamide in patients with advanced prostate cancer with non-castration testosterone levels. BMC Cancer 2019;19:487.

21. Matsubara N, Chi KN, Özgüroğlu M, et al. Correlation of Prostate-specific Antigen Kinetics with Overall Survival and Radiological Progression-free Survival in Metastatic Castration-sensitive Prostate Cancer Treated with Abiraterone Acetate plus Prednisone or Placebos Added to Androgen Deprivation Therapy: Post Hoc Analysis of Phase 3 LATITUDE Study. Eur Urol 2020;77:494-500.

22. Shipley WU, Seiferheld W, Lukka HR, et al. Radiation with or without Antiandrogen Therapy in Recurrent Prostate Cancer. N Engl J Med 2017;376:417-28.

23. Carrie C, Magné N, Burban-Provost P, et al. Short-term androgen deprivation therapy combined with radiotherapy as salvage treatment after radical prostatectomy for prostate cancer (GETUG-AFU 16): a 112-month follow-up of a phase 3, randomised trial. Lancet Oncol 2019;20:1740-9.

24. Pollack A, Karrison T, Balogh AG, et al. MP72-01 Improvements in freedom from progression with short term androgen deprivation therapy and pelvic lymph node treatment added to prostate bed salvage radiotherapy: the NRG oncology/RTOG 0534 SPPORT trial. J Urol 2019. Available online: https://www.auajournals.org/doi/ pdf/10.1097/01.JU.0000557133.04919.da

25. Ghashghaei M, Niazi TM, Heravi M, et al. Enhanced radiosensitization of enzalutamide via schedule dependent administration to androgen-sensitive prostate cancer cells. Prostate 2018;78:64-75.

26. Tombal B, Borre M, Rathenborg P, et al. Long-term Efficacy and Safety of Enzalutamide Monotherapy in Hormone-naïve Prostate Cancer: 1- and 2-Year Openlabel Follow-up Results. Eur Urol 2015;68:787-94.

27. Daniell HW, Dunn SR, Ferguson DW, et al. Progressive osteoporosis during androgen deprivation therapy for prostate cancer. J Urol 2000;163:181-6.

28. Smith MR, McGovern FJ, Zietman AL, et al. Pamidronate to prevent bone loss during androgen-deprivation therapy for prostate cancer. N Engl J Med 2001;345:948-55.

29. Crook JM, O'Callaghan CJ, Duncan G, et al. Intermittent androgen suppression for rising PSA level after radiotherapy. N Engl J Med 2012;367:895-903. 
30. Smith MR, Lee H, Nathan DM. Insulin sensitivity during combined androgen blockade for prostate cancer. J Clin Endocrinol Metab 2006;91:1305-8.

31. Smith MR, Finkelstein JS, McGovern FJ, et al. Changes in body composition during androgen deprivation therapy for prostate cancer. J Clin Endocrinol Metab 2002;87:599-603.

32. Klotz L, Loblaw A, Siemens R, et al. A Phase II, Randomized, Multicenter Study Comparing 10 Months versus 4 Months of Degarelix Therapy in Prolonging the Off Treatment Interval in Men with Localized Prostate

Cite this article as: Lyou Y, Dorff TB. Hormonal manipulation in androgen signaling: a narrative review on using novel androgen therapy agents to optimize clinical outcomes and minimize side effects for prostate cancer patients. Transl Androl Urol 2021;10(7):3199-3207. doi: 10.21037/tau-20-1053
Cancer Receiving Intermittent Androgen Deprivation Therapy for Biochemical Recurrence following Radical Local Therapy. J Urol 2018;200:335-43.

33. Fizazi K, Shore N, Tammela TL, et al. Darolutamide in Nonmetastatic, Castration-Resistant Prostate Cancer. N Engl J Med 2019;380:1235-46.

34. Moilanen AM, Riikonen R, Oksala R, et al. Discovery of ODM-201, a new-generation androgen receptor inhibitor targeting resistance mechanisms to androgen signaling-directed prostate cancer therapies. Sci Rep 2015;5:12007. 